hep-th/0205108

SU-ITP-02/16

SLAC-PUB-9205

TIFR-TH/02-17

\title{
Supersymmetry Changing Bubbles in String Theory
}

\author{
Shamit Kachru ${ }^{a 1}$, Xiao Liu ${ }^{a 2}$, Michael Schulz ${ }^{a 3}$ and Sandip P. Trivedi ${ }^{b 4}$ \\ ${ }^{a}$ Department of Physics and SLAC, Stanford University \\ Stanford, CA 94305/94309, USA \\ ${ }^{b}$ Tata Institute of Fundamental Research \\ Homi Bhabha Road, Mumbai 400 005, INDIA
}

\begin{abstract}
We give examples of string compactifications to $4 \mathrm{~d}$ Minkowski space with different amounts of supersymmetry that can be connected by spherical domain walls. The tension of these domain walls is tunably lower than the $4 \mathrm{~d}$ Planck scale. The "stringy" description of these walls is known in terms of certain configurations of wrapped Dirichlet and NS branes. This construction allows us to connect a variety of vacua with $4 \mathrm{~d} \mathcal{N}=4,3,2,1$ supersymmetry.
\end{abstract}

May 2002

\footnotetext{
1 skachru@stanford.edu

2 liuxiao@stanford.edu

3 mschulz@stanford.edu

4 sandip@tifr.res.in
} 


\section{Introduction}

String theory is known to have many different vacua. An important direction of research aims at understanding whether these different vacua are connected. For compactifications to 4 dimensional Minkowski space the situation is as follows. With $\mathcal{N}=4$ supersymmetry (susy), it is known that there are several disconnected components of the space of vacua (see e.g. [1]). With $\mathcal{N}=2$ susy, naively disconnected components are known to be connected up in a large web [2], although it is premature to say that all such models are connected. For $\mathcal{N}=1$, less is known,1 although some classical obstructions to connecting vacua are circumvented by string theory via chirality changing phase transitions [3].

In the discussion above, the notion of connectedness relates to moving along a moduli space of degenerate solutions. It is known that Minkowski vacua with different amounts of susy can never be connected, in this sense. A theorem to this effect was proved for the perturbative heterotic theory in [4]. However, it is clear that weaker notions of connectedness exist and could be physically relevant. For instance, two vacua can be connected by a finite potential barrier, $V_{\text {bar }}$. For $V_{\text {bar }}$ much less than the four dimensional (4d) Planck scale $M_{4}$, low-energy field theory would correctly describe the dynamics in rolling betwen these vacua. Such a notion of connectedness might be relevant in cosmology. A related weaker notion of connectedness requires the existence of vacuum bubbles of one vacuum inside the other, with the domain wall separating the two having a tension $\sigma \ll\left(M_{4}\right)^{3}$.

In this letter we show that one can unify some vacua with different amounts of supersymmetry in this weaker sense. Our starting point is IIB string theory compactified on the $T^{6} / Z_{2}$ orientifold. This vacuum has $\mathcal{N}=4$ susy and is a dual description of the heterotic theory on $T^{6}$. Appropriately turning on RR and NS fluxes yields vacua with $\mathcal{N}=3,2,1$ susy [5,6,7]. We show that the vacua with reduced susy can be connected to the $\mathcal{N}=4$ vacuum, and to each other, by spherical domain walls. In the ten dimensional string theory, these domain walls are made up of NS and Dirichlet five branes, each of which wrap an internal three cycle, besides spanning the spherical boundary. It is important to note that the tension of the resulting domain walls can be made parametrically lighter than $\left(M_{4}\right)^{3}$ (by tuning the compactification volume $V$, as we will demonstrate). This ensures

1 In this case, we generically expect the moduli to be lifted by quantum corrections. For this reason our discussion will mostly focus on $\mathcal{N} \geq 2$ vacua, but our idea would also apply to any $\mathcal{N}=1$ models where the flux-generated no-scale potential is the full potential. 
that the vacuum inside the bubble is not shielded from the one outside by a black hole horizon, and is available for inspection from the outside.

The bubble configuration we construct is not BPS and evolves in time, with a trajectory determined primarily by the tension of the domain wall. By tuning the radius of the internal space, one can make the lifetime of the bubble arbitrarily large.

We should emphasise that the vacua under consideration here are quantum mechanically stable, and all of them have zero ground state energy. As a result, the spherical bubbles referred to above are not produced by quantum tunneling, as in the decay of a false vacuum.

In $\S 2$, we briefly review the construction of the various vacua in IIB on $T^{6} / Z_{2}$ with fluxes. $\S 3$ describes the domain wall brane configurations which interpolate between the different vacua. We discuss the construction of the domain walls from wrapped five branes, their resulting dynamics, and the stability of the walls. As a concrete example we consider a bubble of the standard $\mathcal{N}=4$ vacuum inside a theory with $\mathcal{N}=2$ susy. We close with a discussion in $\S 4$.

\section{Vacua with various $\mathcal{N}$ in IIB on $T^{6} / Z_{2}$}

Our starting point is IIB theory compactified on a $T^{6} / Z_{2}$ orientifold. This model is T-dual to Type I theory and preserves $\mathcal{N}=4$ supersymmetry. 16 D3 branes are needed to cancel the RR tadpoles arising from the O3 planes. The resulting low energy theory is $S O(32) \mathcal{N}=4$ supersymmetric Yang-Mills theory coupled to $\mathcal{N}=4$ supergravity.

However, this is not the most general possibility. The IIB compactification also admits other superselection sectors in which we turn on quantized fluxes of the three-form field strengths $H$ and $F$ originating from the NS-NS and RR sectors. That is, $H$ and $F$ satisfy the conditions

$$
\frac{1}{(2 \pi)^{2} \alpha^{\prime}} \int_{\gamma} F=m_{\gamma} \in \mathbf{Z}, \quad \frac{1}{(2 \pi)^{2} \alpha^{\prime}} \int_{\gamma} H=n_{\gamma} \in \mathbf{Z},
$$

where $\gamma$ labels the classes in $H_{3}\left(T^{6}, \mathbf{Z}\right)$. For the case of a six-torus with coordinates $x^{i}$ and $y^{i}$, each of period 1 , we can be very explicit about this choice. Let $d \xi^{a}=d x^{i}, d y^{j}, 1 \leq$ $i, j \leq 3$, denote six one-forms. Then, a basis for $H^{3}\left(T^{6}, \mathbf{Z}\right)$ is given by the twenty three

forms, $d \xi^{a} \wedge d \xi^{b} \wedge d \xi^{c}, 1 \leq a, b, c \leq 6$. For the most general choice of flux, $\frac{1}{(2 \pi)^{2} \alpha^{\prime}} F$ and, $\frac{1}{(2 \pi)^{2} \alpha^{\prime}} H$ can be expanded in this basis with integer coefficients. 
In the presence of such fluxes, the full tadpole cancellation condition for the D3 brane charge reads: ${ }^{2}$

$$
\frac{1}{2(2 \pi)^{4}\left(\alpha^{\prime}\right)^{2}} \int_{T^{6}} H \wedge F+N_{D 3}=16 .
$$

Here we consider only the susy preserving case with no anti-branes. Susy breaking by adding anti-branes and vacuum bubbles in similar backgrounds was studied in [8].

In sectors with non-vanishing flux, one finds an effective (super)potential for the Calabi-Yau complex structure and Kähler moduli [9] (for a detailed derivation, see Appendix A of [10]). Supersymmetric vacua are located at points in complex structure moduli space where $G=F-\phi H$ is of type $(2,1)$ (here $\phi$ is the IIB axio-dilaton), while the Kähler structure $J$ should be chosen to make $G$ primitive (i.e. satisfy $J \wedge G=0$ ). These conditions were studied in detail for the case of $T^{6} / Z_{2}$ in [6], and it was found that for generic choices of the fluxes there are no supersymmetric critical points. However, for suitable non-generic choices of flux, one can find vacua with $\mathcal{N}=1,2,3$ supersymmetry. In these vacua, typically all the complex structure moduli and some of the Kähler moduli are fixed. The dilaton-axion is also typically fixed with $g_{s} \sim O(1)$. One Kähler modulus, governing the overall volume of compactification $V$, is never lifted in these models; this will be important in the discussion below.

In $\S 3.3$, a specific $\mathcal{N}=2$ vacuum will be considered. It corresponds to the choice of flux

$$
\begin{aligned}
& \frac{1}{(2 \pi)^{2} \alpha^{\prime}} F=2 d x^{1} \wedge d x^{2} \wedge d y^{3}+2 d y^{1} \wedge d y^{2} \wedge d y^{3} \\
& \frac{1}{(2 \pi)^{2} \alpha^{\prime}} H=2 d x^{1} \wedge d x^{2} \wedge d x^{3}+2 d y^{1} \wedge d y^{2} \wedge d x^{3} .
\end{aligned}
$$

Following [5,6] one easily finds that there is a moduli space of $\mathcal{N}=2$ supersymmetric vacua with these fluxes. A particular locus in this moduli space has $\phi=i$ and a $T^{6}$ which is of the form $\left(T^{2}\right)^{3}$, where each two-torus has complex structure $\tau=i$. The Kähler form can be chosen to be $J \sim i R^{2} \sum_{i=1}^{3} d z_{i} \wedge d \bar{z}_{i}$. This is just a product of square two-tori with overall volume $R^{6}$.

A quick way to see that the vacuum preserves $\mathcal{N}=2$ supersymmetry is by noticing that along this locus, $G$ takes the form

$$
\frac{1}{(2 \pi)^{2} \alpha^{\prime}} G=-\frac{i}{2}\left(d z_{1} \wedge d \bar{z}_{2} \wedge d z_{3}+d \bar{z}_{1} \wedge d z_{2} \wedge d z_{3}\right)
$$

$\mathcal{N}=2$ susy requires that there be another inequivalent choice of complex structure which keeps $G$ of type $(2,1)$; this corresponds to taking $z_{1,2} \rightarrow \bar{z}_{1,2}, z_{3} \rightarrow z_{3}$.

2 Here we ignore the possibility of exotic O3 planes and choose the integer coefficients which characterise the flux to be even, as explained in [7]. 


\section{Vacuum bubbles from D5 and NS5 branes}

\subsection{Overview}

The key idea in our construction of bubbles is the following: by wrapping D5/NS5 branes on three cycles of the compact manifold it is possible to construct domain walls in $R^{3,1}$ across which the quantised fluxes in the compact manifold jump. E.g., wrapping a D5 brane on a three cycle causes the flux of $F$ through the dual three cycle to jump by one unit. Since the vacua reviewed above differ essentially in the RR and NS fluxes along the compact directions, this allows different vacua to be connected.

In fact this idea was used in [9] to construct BPS domain walls between $\mathcal{N}=1$ vacua in the setting of non-compact Calabi-Yau constructions. Our interest is in compact internal manifolds, resulting in flat $4 \mathrm{~d}$ spacetime. In this case, we do not expect BPS domain walls to interpolate between vacua with different amounts of supersymmetry for two reasons. First, the central extensions of the supersymmetry algebra do not admit BPS domain walls of nonzero tension between supersymmetric Minkowski vacua in supergravity (see e.g. [11, 12]). 3 Second, planar domain walls have codimension one and are often singular in supergravity, see e.g. [13].

With this in mind, we construct non-BPS spherical domain walls in $R^{3,1}$, separating a bubble of one vacuum inside the wall from another vacuum outside. Two requirements must be met by the domain wall to consistently interpolate between the vacua. First, the flux of $F, H$ must jump appropriately across the wall. Second, the moduli must vary smoothly across it. It is clear that any jump in $F, H$ fluxes can be engineered by choosing D5,NS5 branes wrapping three cycles in the appropriate homology classes. We will choose the minimum area three cycle in each homology class which is consistent with our boundary conditions. The domain wall is then the composite configuration made out of the resulting D5,NS5 branes.

To meet the condition on the moduli, we restrict ourselves here to considering pairs of vacua such that moduli lifted in both vacua are fixed to the same values. The remaining moduli, unfixed in one or both vacua, can then simply be tuned to take the same values on both sides of the wall (we will show that the backreaction of the walls is small enough to make this a good approximation).

3 This is not true in global supersymmetry. The additional constraint in supergravity arises roughly because one needs the superpotential $W$ to vanish for a Minkowski vacuum. 
In fact, this condition is not very restrictive, and allows our construction to connect several vacua, including many with different susy's. For example, since none of the moduli in the standard $\mathcal{N}=4$ vacuum are fixed, it can be connected to all the other vacua in the above manner. This is enough to establish that all the vacua of $\S 2$ are connected by the above construction.

The two vacua connected by the wall will in general have different numbers of D3 branes (2.2). One can verify that the extra D3 branes in one vacuum terminate on the 5 branes making up the domain wall consistently [14. Also, we note that being composed of 5 branes, the resulting domain walls have a thickness of order the string scale. As a result, in analysing their dynamics below we can work in the thin wall approximation.

Some features of the resulting domain wall dynamics were discussed in the introduction. Let us verify that the tension of the domain wall, compared to $\left(M_{4}\right)^{3}$, can be lowered by tuning the volume modulus $V \sim R^{6}$. In the estimate below, we set $g_{s} \sim O(1)$. A 5 -brane wrapping a three cycle of size $R^{3}$ gives rise to a domain wall tension

$$
\sigma \sim R^{3} /\left(\alpha^{\prime}\right)^{3}
$$

On the other hand, $M_{4} \sim\left(\alpha^{\prime}\right)^{-2} R^{3}$, so that $\sigma /\left(M_{4}\right)^{3} \sim\left(\alpha^{\prime}\right)^{3} / R^{6}$. This ratio can be made small by taking $R$ to be large 1

The rest of this section is organised as follows. The time dependent dynamics of the domain wall is analysed in $§ 3.2$, under the assumption that the the wall moves as a single cohesive unit, driven primarily by its net tension. A specific example of two vacua and the interpolating domain wall is discussed next, in $\S 3.3$. Finally in $\S 3.4$, the relative forces between branes which make up the wall are analysed. These forces are found to be small, thereby justifying the analysis of $\S 3.2$.

\subsection{Bubble dynamics}

We begin by neglecting the backreaction of the domain wall on the metric and other closed string modes, and analyse its trajectory in flat space. Next, we estimate the backreaction effects and show that they are small most of the time. All along we work with walls of tension $\sigma \ll\left(M_{4}\right)^{3}$.

4 The tension of the domain wall depends on both the volume and the moduli that control the sizes of the relevant three cycles. We will show that the backreaction of the wall on all moduli, including these, is small. 
A spherical domain wall in flat space is described by the action,

$$
S=-\int_{t_{i}}^{t_{f}} d t 4 \pi \sigma \rho^{2} \sqrt{1-\dot{\rho}^{2}}
$$

or equivalently an energy

$$
M=\frac{4 \pi \sigma \rho^{2}}{\sqrt{1-\dot{\rho}^{2}}} .
$$

The dynamics is easy to work out in detail. For fixed $M$ and initial outward radial velocity, the bubble expands to a maximum size

$$
4 \pi \sigma \rho_{\max }^{2}=M
$$

then recollapses.

Birkhoff's theorem tells us that the spherically symmetric geometry outside the wall is described by the Schwarzschild metric, while that inside the wall is described by flat space. The Schwarzschild radius $R_{s} \sim G_{N} M$ (with $G_{N} \sim M_{4}^{-2}$ the 4 d Newton's constant). The gravitational backreaction is therefore small as long as

$$
\rho \gg G_{N} M
$$

When $\sigma /\left(M_{4}\right)^{3} \ll 1$, (3.5) can be met by suitably choosing the initial radius $\rho_{i}$ and the total energy of the wall. E.g., for a slowly moving wall, $\dot{\rho} \ll 1$, (3.5) is met by taking,

$$
\rho_{i} \ll \frac{\left(M_{4}\right)^{2}}{\sigma} \sim \frac{R^{3}}{\alpha^{\prime}}
$$

where we have used (3.3) and (3.1). Ultimately, as the bubble recollapses, (3.5) will no longer hold and the gravitational backreaction will get significant, potentially leading to the formation of a black hole.6

The important thing to emphasise is that even if a black hole eventually forms, by tuning the volume and other moduli, the time for which the wall lies outside the black hole horizon can be made as large as one wishes. E.g., the time it takes starting from an initial radius $\rho_{i} \leq \frac{R^{3}}{\alpha^{\prime}}$ to recollapse back to $\rho_{f}=\rho_{i}$ is of order $\Delta t \sim \frac{R^{3}}{\alpha^{\prime}}$.

5 We do not consider trajectories where the wall moves in the internal directions. This is a consistent approximation to make.

6 See however the discussion of stability in $\S 3.4$ 
The domain wall also acts as a source for the various moduli that determine its tension. We now show that the back reaction on these moduli is also small as long as the domain wall is well outside its Schwarzschild radius. We denote the canonically normalised modulus under consideration as $\psi$, and by an additive shift ensure that asymptotically far away, $r \rightarrow \infty, \psi=0$ (e.g. for the radius $\psi \sim\left(\log (R)-\log \left(R_{\infty}\right)\right)$. One can show that $\psi$ satsifies the equation:

$$
\nabla^{2} \psi=\frac{\beta \sigma}{\left(M_{4}\right)^{2}} \sqrt{1-\dot{\rho}(t)^{2}} \delta(r-\rho(t))
$$

The right hand term arises because the tension, $\sigma$, depends on $\psi$. $\beta$ is determined by this dependence, and $\rho(t)$ is the radius of the wall.

Since our main concern is the part of the trajectory where the bubble is well outside its Schwarzschild radius, we consider a simplified model for the domain wall's history below. We assume the wall is constructed at time $t=t_{i}$ and then evolves till $t=t_{f}$ with the radius, $\rho(t)$, meeting the condition (3.5) all along. At time $t_{f}$ we assume the bubble is destroyed.

In this example, $\psi$ satisfies the following boundary conditions: 1 it vanishes as $r \rightarrow \infty$ for all $t$, and also as $t \rightarrow \pm \infty$ for all $r$. Also, we choose boundary conditions such that $\psi=0$ inside the bubble.

The resulting solution for $\psi$ is,

$$
\psi=\frac{f_{+}(t+r)+f_{-}(t-r)}{r} .
$$

$f_{ \pm}$meet two junction conditions across the wall: $f_{+}(t+\rho(t))+f_{-}(t-\rho(t))=0$, and, $f_{+}^{\prime}(t+\rho(t))+f_{-}^{\prime}(t-\rho(t))=\frac{\beta \sigma_{0} \rho(t)}{M_{4}^{2}}$, with prime indicating derivative with respect to argument. $\sigma_{0}$ is the tension at $\psi=0$. Using these, we can solve for $f_{-}$in terms of the trajectory $\rho(t)$ :

$$
f_{-}(t-\rho(t))=\left\{\begin{array}{cc}
0 & t<t_{i} \\
\frac{\beta \sigma_{0}}{2 M_{4}^{2}} \int_{t_{i}}^{t} d t \rho(t)\left(1-\dot{\rho}(t)^{2}\right) & t_{i}<t<t_{f} \\
\frac{\beta \sigma_{0}}{2 M_{4}^{2}} \int_{t_{i}}^{t_{f}} d t \rho(t)\left(1-\dot{\rho}(t)^{2}\right) & t_{f}<t
\end{array}\right.
$$

$f_{+}$, and finally $\psi$ can then be determined from (3.8) and the junction conditions above. A small backreaction means $\psi \ll 1$. It is easy to see from (3.9), (3.8) that this requirement is met when the bubble radius is much larger than the Schwarzschild radius, (3.5).

$7 r, t$ are the usual radial and time coordinates in flat $4 \mathrm{~d}$ space. 


\subsection{An Example}

As a concrete example, consider a spherical bubble of the standard $\mathcal{N}=4$ vacuum inside the $\mathcal{N}=2$ vacuum determined by (2.3).

The domain wall in this case consists of two kinds of D5 branes and two kinds of NS5 branes. The D5 branes wrap the three cycles $x_{1}=x_{2}=y_{3}=0$ and $y_{1}=y_{2}=y_{3}=0$, respectively, with appropriate orientations. Each of these branes carries two units of D5 brane charge. The NS5 branes, each carrying two units of NS 5-charge, wrap the three cycles $x_{1}=x_{2}=x_{3}=0$ and $y_{1}=y_{2}=x_{3}=0$ respectively.

The compactification also has $64 \mathrm{O} 3$ planes. Finally, the $\mathcal{N}=4$ vacuum has 16 D3 branes, while the $\mathcal{N}=2$ vacuum has 12 D3 branes (2.2). The extra D3 branes in the $\mathcal{N}=4$ vacuum terminate on the 5 branes.

\subsection{Stability}

Our discussion of the wall dynamics assumed that the different branes making up the wall do not come apart due to relative forces between them. This assumption is worth examining, since the configuration breaks supersymmetry and $g_{s} \sim O(1)$ in these vacua.

To begin, it is useful to understand the two sources of susy breaking in this configuration. First, there is the curvature of the two sphere in spacetime, which via the bubble tension gives rise to collective motion of the branes. Second, there is the presence of both the branes, and the three-form flux.

To understand the second source, it is helpful to study the example of $\S 3.3$. Here, we take the decompactification limit, $R \rightarrow \infty$, and consider planar parallel branes in $R^{3,1}$ in this limit (while keeping the orientation of the branes in the internal directions unchanged). The spinor conditions can be analysed as in [15],16]. One finds that the configuration of branes and $\mathrm{O} 3$ planes of $\S 3.3$ preserves $\mathcal{N}=1$ susy, i.e. four supercharges. Also, it turns out that any two components, e.g., two kinds of branes or one brane and the O3 planes, preserve $\mathcal{N}=2$ susy. Breaking to $\mathcal{N}=1$ requires three kinds of branes/planes.

As $R \rightarrow \infty$, the effect of the flux $G$ vanishes and can be neglected. But for finite $R$, the $G$ flux (2.3) contributes additional terms in the spinor equations [16, 17]. Now it turns

8 Essentially the same analysis of susy breaking and stability applies to the domain wall obtained by replacing the $\mathcal{N}=2$ vacuum $(2.3)$ with the example in $\S 7.1$ of [6]. This latter $\mathcal{N}=2$ vacuum lifts all complex structure moduli. 
out that the spinor conditions imposed by the brane configuration are in conflict with those imposed by the fluxes. As a result, supersymmetry is completely broken at finite $R$.

With this example in mind, let us return to the general discussion about relative forces between branes. These are of two kinds. First, the different branes couple to different ambient fluxes (the effect of the flux sourced by a brane should be neglected in this interaction). The electric potential energy of a brane in the flux background is

$$
V_{\text {flux }}(\rho) \sim \mu \int C_{(6)} \sim\left(\alpha^{\prime}\right)^{-2} \rho^{3}
$$

where $C_{6}$ is the appropriate gauge potential and we have set $g_{s} \sim O(1)$. $\mu$ is order one in string units. The energy in the tension, (3.1), is $V_{\text {tension }} \sim \sigma \rho^{2} \sim R^{3} \rho^{2} /\left(\alpha^{\prime}\right)^{3}$. Comparing, we see that

$$
V_{\text {flux }}(\rho) / V_{\text {tension }}(\rho) \sim \alpha^{\prime} \rho / R^{3} \text {. }
$$

This ratio is small as long as the bubble radius is bigger than the Schwarzschild radius, (3.5).

Second, interbrane forces could arise if in the absence of flux, the brane/plane configuration breaks susy completely, or, as in the example above, the brane/plane configuration preserves only $\mathcal{N}=1$ susy, which allows for a superpotential to be generated. One expects the resulting (super)potential to scale like the common world volume of the branes required to reduce the susy to $\mathcal{N} \leq 1$. To be comparable with $V_{\text {tension }}$ the potential must scale like $R^{3}$, so all the required branes must be parallel in the internal direction. 0 N $\leq 1$ susy then leaves only one possibility: a pair of NS and D5 branes. Such a pair of 5 branes breaks all susy's. However, in this case the pair can be replaced by a 5 brane susy preserving bound state carrying both NS and D5 brane charge, which results in the same jump in $F, H$ flux. Thus, by appropriately choosing the components of the domain wall, such forces can be made small.

9 In $§ 3.3$ three branes/planes are neccessary to break susy to $\mathcal{N}=1$, and the common world volume lies entirely in the $R^{3,1}$ spacetime. Thus the resulting potential energy $\delta V$ scales like $\delta V \sim \rho^{2} /\left(\alpha^{\prime}\right)^{3}$ and is small compared to $V_{\text {tension }}$. 


\section{Discussion}

The general idea of unifying vacua through vacuum bubbles of some fixed tension, $\sigma$, was discussed by Banks in [18]. There, the focus was on how gravitational back-reaction makes it difficult to imagine using such bubbles as a diagnostic in gravity theories (as opposed to field theories). In our construction, however, the moduli spaces we are unifying allow us to make $\sigma$ very small in $4 \mathrm{~d}$ Planck units, and hence to manufacture bubbles which are large but are not yet black holes. In such a circumstance, we find the notion of "unification through vacuum bubbles" meaningful, even in the presence of gravity.

Our construction connects vacua with large enough volume. Starting with a vacuum where the volume modulus is small, one can imagine first creating a large region of spacetime where the volume modulus is large. This can be done by a slowly varying, large amplitude wave of the volume modulus. In this region the bubble construction could then proceed as before. While we have not explored such time dependent solutions with moduli waves and spherical bubbles in detail, it seems quite reasonable that they exist. 10

Moving beyond, as a next step in making our construction useful in the overall scheme of string duality, it would be important to find transitions connecting e.g. $\mathcal{N}=2$ models on $T^{6} / Z_{2}$ to $\mathcal{N}=2$ compactifications on some more generic Calabi-Yau space. In such a case, up to standard dualities, one would have successfully unified the heterotic string on $T^{6}$ with the best-understood web of vacua with less supersymmetry.

It would also be interesting to study connectedness by asking if one can find time dependent solutions which roll between vacua separated by a finite potential barrier. The fact that $\sigma \ll M_{4}^{3}$ in our construction is suggestive. However, the existence of such solutions cannot be explored in low energy field theory, since the fields which create the five-branes would also have to be excited. Exploring such solutions in string field theory seems difficult, at the moment.

\section{Acknowledgements}

We would like to thank A. Dabholkar, S. Das, M. Douglas, S. Ferrara, S. Giddings, M. Porrati, S. Shenker, E. Silverstein, L. Susskind, P. Tripathi, H. Verlinde, E. Witten and especially T. Banks for helpful and encouraging discussions. This work was supported

10 A similar construction should also apply to directly connect two vacua in which some of the moduli are fixed to different values. The fluxes give rise to a potential on moduli space, $V_{\text {pot }} /\left(M_{4}\right)^{4} \sim\left(\alpha^{\prime}\right)^{6} / R^{12}$, which is small for large $R$. Hence, the resulting bubble should still be accessible from the outside vacuum. 
in part by the Department of Energy under contract DE-AC03-76SF00515. The work of S.K. is also supported by a Packard Fellowship, a Sloan Fellowship, and NSF grant PHY-0097915. 


\section{References}

[1] J. de Boer, R. Dijkgraaf, K. Hori, A. Keurentjes, J. Morgan, D. R. Morrison and S. Sethi, Adv. Theor. Math. Phys. 4, 995 (2002) arXiv:hep-th/0103170].

[2] B. Greene, D. Morrison and A. Strominger, Nucl. Phys. B451, 109 (1995) arXiv:hepth/9504145;

A. Avram, P. Candelas, D. Jancic and M. Mandelberg, Nucl. Phys. B465, 458 (1996) arXiv:hep-th/9511230;

T. Chiang, B. Greene and M. Gross, Nucl. Phys. Proc. Suppl. 46, 82 (1996) arXiv:hepth/9511204.

[3] S. Kachru and E. Silverstein, Nucl. Phys. B504, 272 (1997) [arXiv:hep-th/9704185;

B. Ovrut, T. Pantev and J. Park, JHEP 0005, 045 (2000) arXiv:hep-th/0001133.

[4] T. Banks and L. Dixon, Nucl. Phys. B307, 93 (1988).

[5] K. Dasgupta, G. Rajesh and S. Sethi, JHEP 9908, 023 (1999) arXiv:hep-th/9908088.

[6] S. Kachru, M. Schulz and S. Trivedi, [arXiv:hep-th/0201028].

[7] A. Frey and J. Polchinski, [arXiv:hep-th/0201029].

[8] S. Kachru, J. Pearson and H. Verlinde, arXiv:hep-th/0112197.

[9] S. Gukov, C. Vafa and E. Witten, Nucl. Phys. B584, 69 (2000) arXiv:hepth/9906070|.

[10] S. B. Giddings, S. Kachru and J. Polchinski, arXiv:hep-th/0105097.

[11] M. Cvetic and H. Soleng, Phys. Rept. 282, 159 (1997) [arXiv:hep-th/9604090.

[12] S. Ferrara and M. Porrati, Phys. Lett. B423, 255 (1998) arXiv:hep-th/9711116.

[13] J. Gutowski, Nucl. Phys. B627, 381 (2002) arXiv:hep-th/0109126.

[14] A. Strominger, Phys. Lett. B383, 44 (1996) [arXiv:hep-th/9602111.

[15] A. Hanany and E. Witten, Nucl. Phys. B492, 152 (1997) [arXiv:hep-th/9611230.

[16] M. Grana and J. Polchinski, arXiv:hep-th/0106014.

[17] K. Becker and M. Becker, Nucl. Phys. B477, 155 (1997) arXiv:hep-th/9605053

[18] T. Banks, arXiv:hep-th/0011255. 